\title{
A SENSITIVE RESONANT GAS SENSOR BASED ON MULTIMODE EXCITATION OF A BUCKLED BEAM
}

\author{
Amal Z. Hajjaj ${ }^{\ddagger, 1}$, Nizar Jaber, ${ }^{\ddagger 1,2}$, Nouha Alcheikh ${ }^{1}$, and Mohammad I. Younis ${ }^{1, *}$ \\ ${ }^{1}$ Physical Sciences and Engineering Division, King Abdullah University of Science and Technology, \\ Thuwal, 23955-9600, Saudi Arabia \\ ${ }^{2}$ School of Mechanical Engineering, Purdue University, West Lafayette, IN, 47906, USA.
}

\begin{abstract}
We report a new gas sensing technique based on the simultaneous tracking of multiple modes of vibration of an electrothermally heated bridge resonator operated near the buckling point. The proposed technique maximizes the sensitivity of the sensor to the change in the gas concentration and enables the identification of the gas type without the need for selective coating. Under the same gas concentration value, the reported measurements demonstrate a $200 \%$ change in the frequency in contrast to $0.5 \%$ using the conventional technique based on resistance measurements. The proposed method is simple in principle and design and is promising for achieving practical low-cost gas sensors.
\end{abstract}

\section{KEYWORDS}

Gas sensor, thermal conductivity, bridge resonator, buckling point, electrothermally heated.

\section{INTRODUCTION}

The quest for ultra-sensitive low-cost miniaturized gas sensors in the past few decades has sparked interest to seek alternative techniques other than the conventional gas sensors which require large surface areas and special coating materials for selective and sensitive detection. Functionalization is realized by coating the surface of the MEMS gas sensor with a thin layer of material that have affinity for particular gases, such as gold for mercury detection [1], polymer doped with carbon nanotube to detect Carbon dioxide [2], palladium for Hydrogen sensing [3], and metal-organic frameworks for humidity and volatile organic compounds $[4,5]$. The performance of such devices depends on the functionalization type, thickness, and the sensor design.

MEMS gas sensors based on thermal conductivity measurements [6-9] have been known to be among the promising candidates. These sensors rely on thermal energy dissipation (cooling or heating) of a heated structure due to the alteration of the surrounding gas concentration, which alleviates the requirement of large surface area and special techniques to functionalize the sensor. The temperature of the heated structure is altered from the surrounding gas properties; mainly its effective thermal conductivity. These sensors are fabricated from materials with high temperature coefficient of resistance, which enables sensitive measurement of the bridge temperature by tracking the resistance. In recent studies $[6,7]$, MEMS heated bridges have been employed to detect noble gases and demonstrated promising results for binary gas mixtures. These sensors show long lifetime and great stability compared to absorption-based gas sensors.

The thermal conductivity based gas sensors rely on the resistance variation of the heated structures due to gas exposure, which is typically less than few percents. Hence, there is a strong demand for a more sensitive probe to measure the gas concentration.

Monitoring the frequency of MEMS resonators due to gas concentration change has demonstrated ultra-sensitive detection of gas up to the molecule levels. Recently, significant attention has been dedicated to track the resonance frequency of MEMS resonators for gas sensing application [1, 3-5, 10] instead of resistance variation showing higher sensitivities. Moreover, several dynamical features, such as the bifurcation points, internal resonance, and secondary resonances are utilized to improve the sensor sensitivity. In a previous work [11], we exploited the buckling point (bifurcation point) of heated silicon bridge to demonstrate sensitive and scalable pressure sensor. The concept is based on tracking the fundamental frequency of the electrothermally buckled bridge upon changing the surrounding pressure. Raising the surrounding pressure increases the heat dissipation, which reduces the bridge temperature. The cooling effect of the surrounding air changes the overall stress of the structure, and thus its fundamental frequency.

Here, we present a sensitive technique for measuring the gas concentration and identifying the gas type using an electrothermally heated bridge microresonator operated near the buckling point. The method is based on the simultaneous recording of the first and second modes frequencies while changing the gas concentration and type.

\section{EXPERIMENTAL SETUP}

To demonstrate the concept, we utilize an in-plane clamped-clamped microbeam, Fig. 1a, fabricated from a highly conductive $30 \mu \mathrm{m}$ Silicon device layer using the standard surface micromachining process. The process steps are lithography to transfer the device design from the mask to the wafer, metal sputtering to create the connection pads, deep reactive ion etch (DRIE) to form the structure, and vapor hydrofluoric acid dry etch (vpourHF) to release the device. The tested microresonator is of

\footnotetext{
${ }^{\mathrm{t}} \mathrm{N}$. Jaber and A. Z. Hajjaj contributed equally to this work *Corresponding author: Mohammad I. Younis, e-mail: Mohammad.Younis@kaust.edu.sa, ORCID: orcid.org/0000-00029491-1838
} 
length $500 \mu \mathrm{m}$, width $30 \mu \mathrm{m}$, and thickness $2 \mu \mathrm{m}$. The microbeam is separated from a stationary electrode with a transduction gap of width $8 \mu \mathrm{m}$. Here, we use a half electrode configuration to break the symmetry of the excitation force, which enables the excitation of the antisymmetric modes [12].

The experimental setup, shown in Fig. 1, is used to expose the device to different types and levels of gas concentration and track the change in the resonance frequencies. The microbeam is placed in a test chamber, which is equipped with ports to provide the actuation signals. The device is tested under atmospheric pressure and room temperature. The gas setup is composed of two mass flow controllers, which control the flow of the targeted gas and the dry Nitrogen. The ratio of the two flow values determines the gas concentration inside the chamber. We utilize the laser Doppler vibrometer to measure the resonance frequencies of the microresonator due to white noise signal. Also, a separate voltage source, $\mathrm{V}_{\mathrm{Th}}$, is utilized to induce a current flowing through the microbeam, which heats it up by the Joule's heating effect, and hence alters its stiffness and resonance frequencies. Using the LABVIEW software and the LCR meter, we record the real-time variation of the microbeam resistance as we expose it to different gas concentrations.

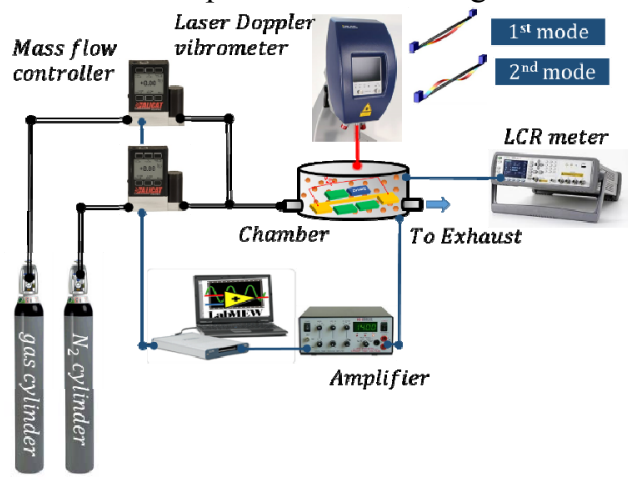

Figure 1: A schematic of the experimental setup.

\section{PRINCIPE OF OPERATION}

Fig. 2 shows the measured frequencies of the first and second mode of vibration at different electrothermal voltages. As shown, increasing the electrothermal voltage, $\mathrm{V}_{\mathrm{Th}}$, reduces the resonance frequency values, which can be attributed to the decrease in the microbeam stiffness due to the induced compressive stress until the buckling point. After buckling, a sudden increase in the first mode is observed due to the increase of stiffness of the buckled microbeam due to the stretching mechanism. The second mode frequency remains constant and is not affected with the stretching due to the pure bending nature of the second mode shape.

Operating the resonator near the buckling point maximizes the sensitivity to variation in the first mode frequency to physical phenomena that affect the axial stress such as cooling and heating effects [11]. Exposing the resonator to gases with higher/lower thermal conductivity compared with Nitrogen reduces/increases the axial stress inside the microbeam, which alters the values of the resonance frequencies. Hence, by tracking the shift in the first mode, we can quantify the concentration of the targeted gas, while by tracking the shift in the second mode, we can identify the type of gas (having higher/lower thermal conductivity comparing to Nitrogen). To demonstrate the concept, we exposed the resonator to Helium (He) and Argon (Ar), which have higher $(0.151 \mathrm{~W} / \mathrm{mK})$ and lower $(0.018 \mathrm{~W} / \mathrm{mK})$ thermal conductivities, respectively, compared to Nitrogen $(0.026 \mathrm{~W} / \mathrm{mK})$.

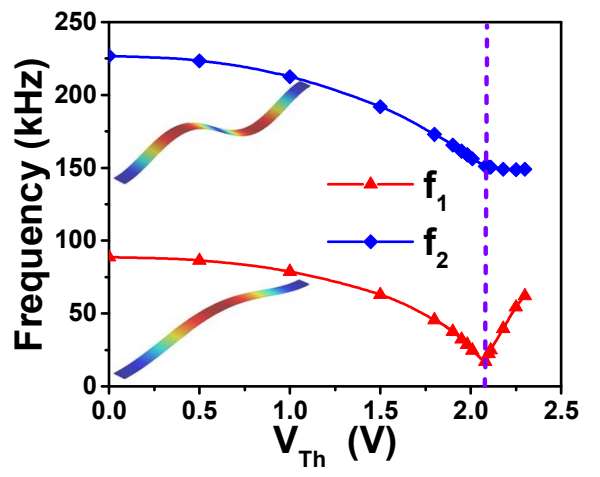

Figure 2. Variation of the first two resonance frequencies of the bridge microresonator with $V_{T h}$. The vertical line shows the operating point (buckling point) of the sensor. Note that at the buckling point the first mode frequency does not reach zero due to the electrostatic bias, which perturbs the symmetric bifurcation [13].

\section{RESULTS AND DISCUSSION}

Next, we tune the electrothermal voltage at the buckling point for all the gas measurements to maximize the proposed sensor sensitivity to any axial change due to the presence of different gases. Firstly, we expose the chamber with different concentrations of Helium while recording the real-time variation of the resistance of the microbeam. As shown in the inset of Fig. 3, increasing in the Helium concentration decreases the resistance of the microbeam. This proves that as the Helium concentration increases, the effective thermal conductivity of the medium surrounding the microbeam increases leading to more convection of the beam surface which reduces the microbeam temperature. The reduction in the temperature reduces the compressive force, which increases the first and second resonance frequencies as shown in Fig. 3. In contrast to the resistance measurements, the frequency values show significant improvement in the sensor response, which shows two orders of magnitude improvement under the same gas concentration value.

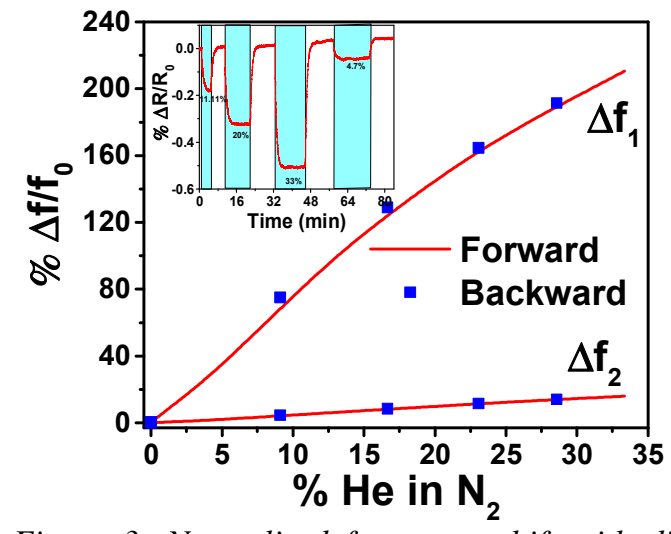

Figure 3: Normalized frequency shift with different percentages of Helium mixed with Nitrogen. The inset 
shows the normalized resistance variation with time for different percent of Helium mixed with Nitrogen. Results are shown when increasing (forward) and decreasing (backward) the gas concentration.

On the other hand, exposing the resonator to Argon increases the resistance of the microbeam, as depicted in the inset of Fig. 4. Increasing the Argon concentration decreases the effective thermal conductivity of the medium surrounding the microbeam, hence, less heat convection from the beam. This increases the temperature, and hence the compressive stress in the microbeam. Increasing the Argon concentration raises the frequency of the first mode while the second mode remains constant. The recorded frequency variation of the first resonance frequency is shown to reach $60 \%$ of the original value, which is significantly larger than the resistance measurements.

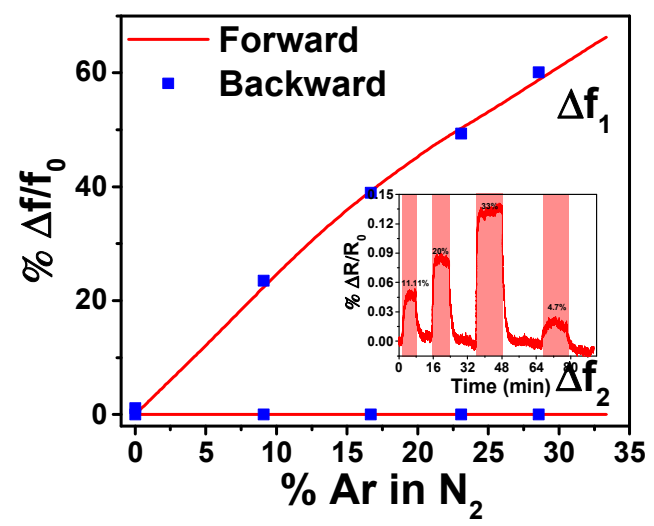

Figure 4: Normalized frequency shift with different percentages of Argon mixed with Nitrogen. The inset shows the normalized resistance variation with time for different percentages of Argon mixed with Nitrogen.

One should note that the operation speed of the proposed gas sensor is limited by the thermal time constant that is around $176 \mu \mathrm{s}$. For faster operation, the sensor needs to be further miniaturized to the nano scale. The sensitivity of the proposed device to temperature variation from the environment is a major factor to be considered. Hence, calibration experiments need to be conducted to overcome the variation of the ambient temperature. Another factor to be considered is the low quality factor of the proposed gas sensor as operated at atmospheric pressure. Accordingly, these concerns need further investigation for the final implementation of the proposed gas sensor.

Detecting harmful gases, such as Hydrogen $\left(\mathrm{H}_{2}\right)$, Methane $\left(\mathrm{CH}_{4}\right)$, Propane $\left(\mathrm{C}_{3} \mathrm{H}_{8}\right)$, and Carbon dioxide $\left(\mathrm{CO}_{2}\right)$, etc., has been the focus of in-depth study in the past few decades. Such gases cause serious environmental and health problems. Thus, we have conducted a multiphysics finite elements study using COMSOL Multiphysics to demonstrate the potential of the proposed technique in detecting such harmful gases.

To take into account the various physical domains, various solvers have been coupled including the Solid Mechanics, Electric Currents, and Heat Transfer interface domains. The model couples the Joules heating of doped polysilicon and convective heat transfer for the surrounding gas. The anchors of the bridge resonator are assigned a fixed constraint boundary condition with ambient temperature at their bottom. The rest of the faces of the structure are set to a convective heat boundary condition, where the heat flux option is used for external natural convection with the gas mixture (the mixture of Nitrogen and the targeted gas) at ambient temperature and atmospheric pressure. The model takes into account the temperature dependence of the thermal properties of the gas mixture and the polysilicon beam. The effective thermal conductivity of the gas mixture, known to be a nonlinear function of different parameters (thermal conductivity, viscosity, molar mass of each gas), was calculated using the Wassiljewa formula and then implemented in the software $[14,15]$.

The simulated results of the resonance frequencies variation of the bridge microresonator with different gas concentrations, Hydrogen, Methane, Propane, and Carbon dioxide, are shown in Fig. 5. The simulation results prove the same concept presented experimentally and demonstrate the applicability of the proposed concept in different harmful gases without the need for any specific functionalization for each gas. The proposed finite element model can be employed to optimize the sensor design to get higher frequency variation for lower gas concentration.

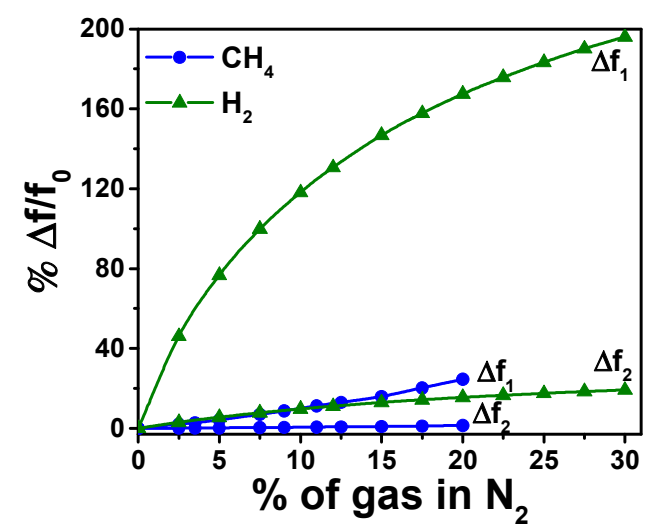

(a)

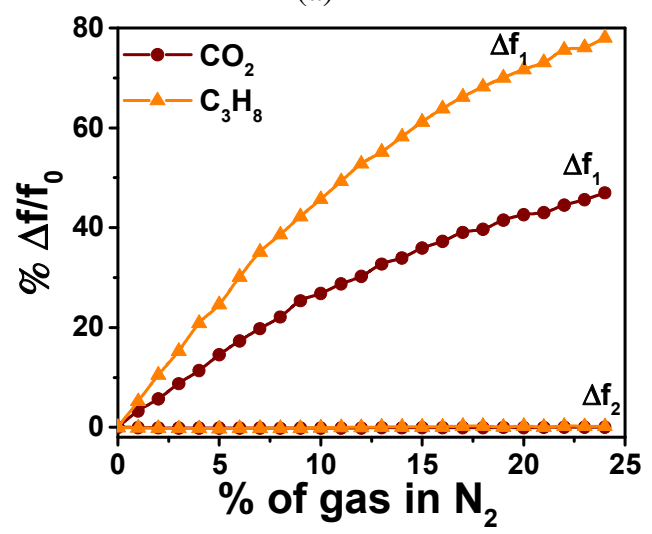

(b)

Figure 5: Simulated normalized frequency shifts with different percentages of various gases mixed with Nitrogen, using the software COMSOL Multiphysics. 


\section{CONCLUSIONS}

We proposed a new sensitive probe for measuring the gas concentration and identifying the gas type using an electrothermally heated bridge operated near the buckling point. The concept is based on the simultaneous tracking of the first and second modes at different gas concentration levels. The frequency values depend on the effective convective cooling/heating of the gas mixture surrounding the heated bridge MEMS resonators. By tracking the shift in the first mode, the concentration of the targeted gas could be quantified. The shift in the second mode is employed to determine the type of gas (having higher/lower effective thermal conductivity compared to Nitrogen). The results demonstrate the possibility of realizing a gas sensor that can determine the type of gas without the need for selective coating. In contrast to resistance-based measurement, operating the bridge near the buckling point and monitoring the frequency shift maximize the sensor sensitivity. Under the same value of gas concentration, the results show a $200 \%$ relative frequency change compared with few percents when tracking the resistance value. The obtained results motivate in-depth studies for the implementation of the proposed sensor in binary and multi gas mixtures. The significant advantage of the proposed scalable gas sensor is the simplicity of fabrication, operating principle, and sensing scheme. The device design can be optimized using finite element analysis to enhance the sensitivity of the proposed sensor, which enables the detection of low concentration of different harmful gases.

\section{ACKNOWLEDGMENTS}

This research was support by KAUST.

\section{REFERENCES}

D. Struk, A. Shirke, A. Mahdavifar, P. J. Hesketh, and J. R. Stetter, "Investigating timeresolved response of micro thermal conductivity sensor under various modes of operation," Sensors and Actuators B: Chemical, vol. 254, pp. 771-777, 2018.

[7] A. Mahdavifar, M. Navaei, P. J. Hesketh, M. Findlay, J. R. Stetter, and G. W. Hunter, "Transient thermal response of micro-thermal conductivity detector $(\mu \mathrm{TCD})$ for the identification of gas mixtures: An ultra-fast and low power method," Microsystems \& Nanoengineering, vol. 1, p. 15025, 2015.

[8] I. Elmi, S. Zampolli, E. Cozzani, F. Mancarella, and G. Cardinali, "Development of ultra-lowpower consumption MOX sensors with ppb-level VOC detection capabilities for emerging applications," Sensors and Actuators B: Chemical, vol. 135, pp. 342-351, 2008.

[9] M. Decarli, L. Lorenzelli, V. Guarnieri, E. Barborini, S. Vinati, C. Ducati, et al., "Integration of a technique for the deposition of nanostructured films with MEMS-based microfabrication technologies: application to micro gas sensors," Microelectronic Engineering, vol. 86, pp. 1247-1249, 2009.

[10] Y. Hwang, H. Sohn, A. Phan, O. M. Yaghi, and R. N. Candler, "Dielectrophoresis-assembled zeolitic imidazolate framework nanoparticlecoupled resonators for highly sensitive and selective gas detection," Nano letters, vol. 13, pp. 5271-5276, 2013.

[11] A. Z. Hajjaj, N. Alcheikh, M. A. A. Hafiz, S. Ilyas, and M. I. Younis, "A scalable pressure sensor based on an electrothermally and electrostatically operated resonator," Applied Physics Letters, vol. 111, p. 223503, 2017.

[12] N. Jaber, A. Ramini, A. A. Carreno, and M. I. Younis, "Higher order modes excitation of electrostatically actuated clamped-clamped microbeams: experimental and analytical investigation," Journal of Micromechanics and Microengineering, vol. 26, p. 025008, 2016.

[13] A. Z. Hajjaj, N. Alcheikh, A. Ramini, M. A. Al Hafiz, and M. I. Younis, "Highly tunable electrothermally and electrostatically actuated resonators," Journal of Microelectromechanical Systems, vol. 25, pp. 440-449, 2016.

[14] S. Udina, M. Carmona, G. Carles, J. Santander, L. Fonseca, and S. Marco, "A micromachined thermoelectric sensor for natural gas analysis: Thermal model and experimental results," Sensors and Actuators B: chemical, vol. 134, pp. 551-558, 2008.

[15] S. Udina, M. Carmona, A. Pardo, C. Calaza, J. Santander, L. Fonseca, et al., "A micromachined thermoelectric sensor for natural gas analysis: Multivariate calibration results," Sensors and Actuators B: Chemical, vol. 166, pp. 338-348, 2012. 